[P1] Naser Tarhuni, Timo Korhonen, Edward Mutafungwa, and M. Elmusrati, "Multi-Class Optical Orthogonal Codes for Multi-Service Optical CDMA Networks", Journal of Lightwave Technology, Vol.24, Issue 2, Feb. 2006, Pages: 694-704. (Reprint from Journal of Lightwave Technology, Copyright (C) (2006), with reprint permission from IEEE).

This material is posted here with permission of the IEEE. Such permission of the IEEE does not in any way imply IEEE endorsement of any of Helsinki University of Technology's products or services. Internal or personal use of this material is permitted. However, permission to reprint/republish this material for advertising or promotional purposes or for creating new collective works for resale or redistribution must be obtained from the IEEE by writing to pubs-permissions@ieee.org.

By choosing to view this document, you agree to all provisions of the copyright laws protecting it. 


\title{
Multiclass Optical Orthogonal Codes for Multiservice Optical CDMA Networks
}

\author{
Naser G. Tarhuni, Student Member, IEEE, Timo O. Korhonen, Member, IEEE, Edward Mutafungwa, and \\ Mohammed S. Elmusrati, Member, IEEE
}

\begin{abstract}
Optical code division multiple access (CDMA)-based networks are an interesting alternative to support various traffic types of multimedia applications with highly variable performance targets. Generally, multilength codes are designed to support multirate services, while the multiweight codes are designed to support differentiated quality of service $(\mathrm{Q} O S)$ for multimedia applications. However, existing optical orthogonal codes (OOCs) are limited to single class or multiclass with restricted weight and length properties. Therefore, there exists a lack of flexibility in the existing OOCs to support arbitrary rate and QoS. This paper presents a proposal of generation procedure and performance analysis of joint multiweight multilength strict OOCs. The approach used in this paper is to apply a methodology strongly relying on developed analytical theory that is supported by computer optimization, because it has turned out that it is mathematically intractable to construct unconstraint joint multilength multiweight OOCs using pure algebraic techniques. The generated code set fulfills the conditions of strictly OOCs, namely, the maximum nonzero shift autocorrelation and the maximum cross correlation constraints of one. The mark position difference (MPD) approach is used to generate in a flexible way the multiclass code set. The MPD results in the simple evaluation of multiclass code set cardinality. Furthermore, the multiple-access interference (MAI) in a multiclass OOC system is evaluated by modeling the interference per class as a Poisson distribution to simplify performance evaluation with acceptable accuracy.
\end{abstract}

Index Terms-Multirate optical CDMA, optical CDMA, optical orthogonal codes.

\section{INTRODUCTION}

$\mathbf{O}$ PTICAL fibers are especially applicable for high-speed local area network (LAN) and related access network applications. New services will mainly be based on the emerging broadband capabilities, with the integration of video and/or multimedia components [1]-[3]. At the user end of the network, passive optical networks (PONs) are being considered as the main technique for the evolving access networks. Access networks (known also as the last mile techniques) linking the high-speed end-user equipment to the high-capacity backbone networks remain a bottleneck. Several medium access techniques in PON have been considered, as for instance widely investigated Ethernet-based PON (EPON) [4]. A PON is a point-to-multipoint optical connection with no active compo-

Manuscript received February 7, 2005; revised May 9, 2005.

N. G. Tarhuni, T. O. Korhonen, and E. Mutafungwa are with the Communications Engineering Laboratory, Helsinki University of Technology, Espoo FIN-02015 HUT, Finland (e-mail: naser.tarhuni@hut.fi; timo.korhonen@hut.fi; edward.mutafungwa@hut.fi).

M. S. Elmusrati is with the Department of Computer Science, University of Vaasa, Vaasa 65101, Finland (e-mail: moel@uwasa.fi).

Digital Object Identifier 10.1109/JLT.2005.862439 nents in the signal path. Connections in the downstream are of broadcast-and-select type. On the other hand, upstream connections from the optical network units (ONUs) at the end user to the optical network terminals (ONTs) at the central office share the same channel. In EPON, each node uses the optical channel for the assigned time slot. To support differentiated quality of service (QoS), EPON employs a central controller that dynamically allocates bandwidth to end users according to the traffic load [5]. Typical user data traffic is bursty by nature; therefore, static channelization (or multiplexing) schemes, realized by permanently allocated time-division multiplexing (TDM) or wavelength-division multiplexing (WDM) slots waste resources for idle users [6]. Users can share the communication bandwidth more effectively by using optical code-division-based techniques. Optical code division multiple access (CDMA) provides more immunity to packet collisions than dynamic bandwidth allocation/time division multiple access (TDMA) approaches, which reduces packet latency and offers improved adaptation to information source statistics.

Fig. 1 shows a schematic diagram of a fiber optic network employing optical code division encoding and decoding. The laser source produces very short (inversely proportional to data rate and code length) optical pulses, which are encoded by using the optical encoder that is an optical finite-impulse response (FIR) filter. The number of encoder taps determines the code weight, which can be controlled by optical switches. At the receiving end, the optical decoder is matched to the desired code with the optical delays equal to the complements of the delays at the encoder (i.e., it is the optical matched filter for the applied code). In intensity-modulated direct detection (IM-DD) systems, the intensity of the optical signal is modulated and detected, and therefore, only unipolar codes can be applied for optical noncoherent multiple access. In [7], optical orthogonal codes (OOCs) were proposed for noncoherent optical CDMA. The OOCs can be generated simply by using optical passive components, such as optical delay lines, optical splitters, and optical combiners [8].

The OOCs are highly sparse codes, and the number of supported users is quite limited. However, the number of users can be increased by using longer codes with lower weights. The use of longer codes requires faster laser sources, which increases fiber dispersion effects, while the lower weights degrade the bit error rate (BER) performance significantly. When suggested for the first time, OOC was proposed with fixed and equal code weights and lengths to satisfy the code correlation properties planned to guarantee a certain number of simultaneous equal data rate and equal error performance users. 


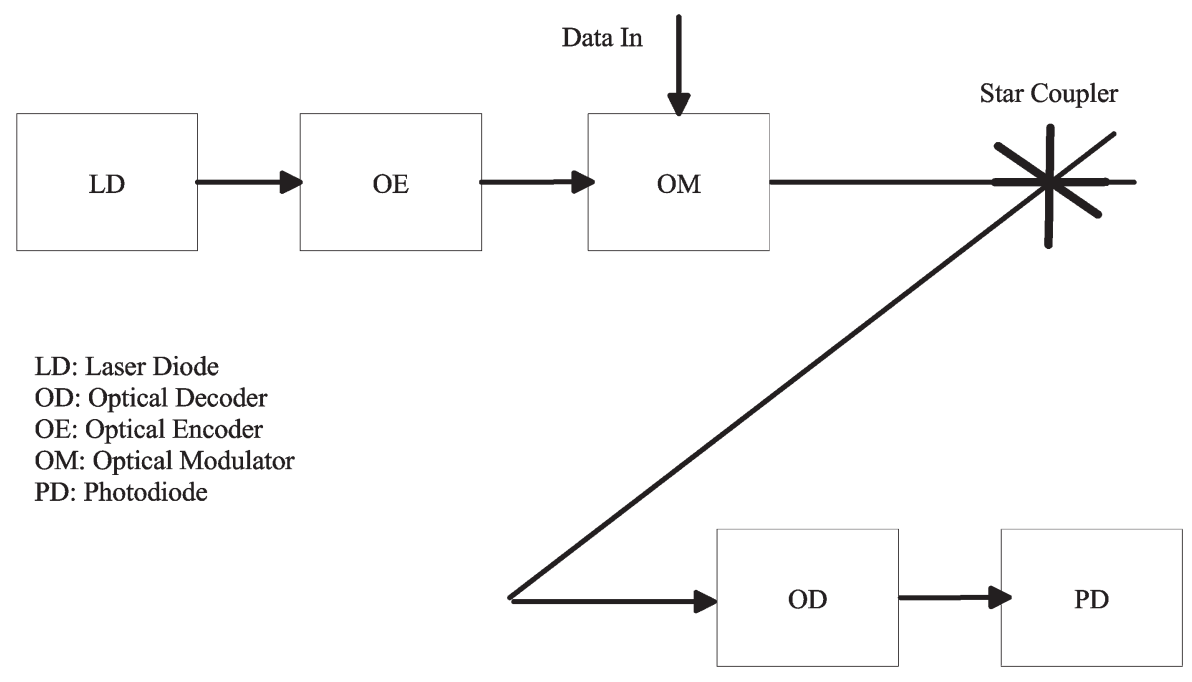

Fig. 1. Optical CDMA network.

Multirate optical CDMA transmission has been investigated in [9]-[11]. In [9], a multirate optical CDMA transmission was achieved by varying the length of the code set whose autocorrelation and cross correlation are constrained to be three or smaller. This limits the system applications due to the relatively high error probability for high rate users. A parallel mapping scheme was applied to multirate optical CDMA in [10]. The scheme is based on assigning a number of code sequences according to the user data rate. This limits the applicability because of the limited number of available codes. Optical fast frequency hopping has been also proposed for multirate optical CDMA based on multiple wavelengths [11]. This is achieved by changing the length of the hopping pattern by using a tunable fiber Bragg grating. Power control was used to improve the system capacity. Disadvantages of this scheme include the need for multiwavelength transmitters and susceptibility to wavelengthdependent impairments.

Kowng and Yang [12] proposed a double-weight twodimensional OOC based on Galois field theory; unfortunately, the proposed OOCs are able to support only two different services without multirate compatibility. Recently, in [13], variable-weight $\mathrm{OOC}$ constructions based on two schemes, balanced pairwise design, and packing design with partition are given. In the second scheme, variable-weight OOCs are constructed by partitioning larger weight codeword into a family of codes with a smaller code weight. On the other hand, multilength OOC was presented in [14] with fixed code weights. The rates of the constructed code set cannot be selected arbitrarily, because short codewords are used to construct longer ones. Moreover, the performance analysis was presented for a double-rate case only. In [15], the variable rate OOC for video applications with fixed weight was proposed. The performance analysis in terms of the BER of the system is not considered. In [16], a two-class OOC was discussed, and the performance of such system was evaluated based on the assumption that the binomially distributed OOC interference is approximated by a Gaussian distribution. This approximation is valid only if the number of users is high and the probability of collision from an interfering user is close to 0.5 . This assumption fails to be accurate in OOCs with long code lengths and low code weights.

In this paper, we introduce the generation and analysis of OOC-based optical network with arbitrary code weights and lengths jointly while preserving the original correlation properties of the OOCs. We call this OOC code set the multiclass OOC or the multiweight multilength OOC (MWML-OOC). The rest of the paper is organized as follows: In Section II, the original OOC code is reviewed and the multiclass OOC is presented. In Section III, we use the single-user conventional detector and summarize the results for the performance analysis of the proposed system. We use the Poisson approximation of the multiple-access interference (MAI) for the single class (a class with OOC codes of the same length and weight) to simplify the evaluation of the multiclass MAI probability distribution and BERs.

\section{MWML-OOC CODE GENERATION}

\section{A. Background}

The OOC code set $\mathbf{C}$ is characterized by the quadruple ( $N, W, \lambda_{a}, \lambda_{c}$ ), where $N, W, \lambda_{a}$, and $\lambda_{c}$ are the code length, code weight, maximum nonzero shift autocorrelation, and maximum cross correlation, respectively [17]. Generally, OOCs are required to satisfy the following correlation properties:

$$
R_{x x}(m)=\sum_{n=0}^{N-1} x_{n} x_{n+m} \leqslant \lambda_{a}
$$

for any $x \in \mathbf{C}$ and any integer $[m]_{\bmod (N)} \neq 0$, where $[m]_{\bmod (N)}$ denotes $m$ modulo $N$, and

$$
R_{x y}(m)=\sum_{n=0}^{N-1} x_{n} y_{n+m} \leqslant \lambda_{c}
$$

for any $(x \neq y) \in \mathbf{C}$ and any integer $m$. The property in (1) makes the synchronization of the intended receiver simpler by constraining the autocorrelation sidelobes to $\lambda_{a}$. On the 


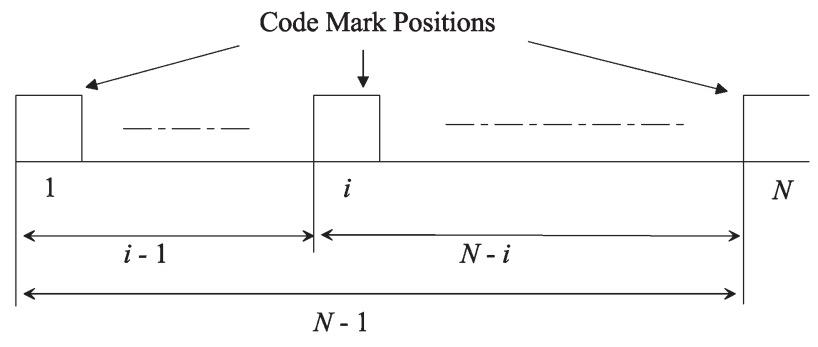

Fig. 2. Mark position differences.

other hand, (2) allows for distinguishing the different users accessing the network simultaneously, meaning that the cross correlations of the users are limited by $\lambda_{c}$. OOC codes with the maximum nonzero shift autocorrelation and the maximum cross correlation bounded by one $\left(\lambda_{a}=\lambda_{c}=1\right)$ are called the strict OOCs [18] and are represented here as $(N, W)$. In [17], several techniques for OOC construction were considered, such as iterative, Greedy, projective geometry, and combinatorial techniques. Furthermore, the upper bound on the number of codes in the strict OOC code set is given by

$$
K \leqslant\left\lfloor\frac{N-1}{W(W-1)}\right\rfloor
$$

where $\lfloor x\rfloor$ is the integer part of $x$.

The difference between chip positions containing marks in the OOC can be any integer number in $\{1,2, \ldots, N-1\}$ (Fig. 2). Associated with each code in the OOC, there is a mark position difference (MPD) set that follows from the principle of incoherent optical delay line decoder operation. (Note that the concept of mark differences have been used in the literature with different names, such as "difference" [7], "extended set" [17], "slot distance" [18], and "adjacent relative cyclic delay" [19].) The elements of MPD set can be evaluated using

$$
\begin{aligned}
d_{n}= & N-\left[N+P_{i}-P_{j}\right]_{\bmod (N)} \\
& \text { for } n=1,2, \ldots, W(W-1) \\
& \text { and }(i \neq j)=1,2, \ldots, W(W-1)
\end{aligned}
$$

where $P_{k}$ is the $k$ th mark position. To satisfy (1) and (2), it is required that there should be no repeated elements in the code MPD and no common elements between the MPDs of any two codes. For instance, consider the two $(150,3)$ OOC codes $c_{1}=[1,61,104]$ and $c_{2}=[1,105,114]$ and the corresponding MPDs following (4): $d_{1}=\{60,103,43,90,47,107\}$ and $d_{2}=$ $\{104,113,9,46,37,141\}$. Since there are no repeated elements in $d_{1}$ and $d_{2}$, and no shared elements between $d_{1}$ and $d_{1}$, the two codes are, therefore, part of a strict OOC code set.

\section{B. $M W M L-O O C$}

In order to support variable data rates and QoSs in optical CDMA network based on OOCs, it is highly desirable to have variable length and variable weight codes. The shorter the codes are, the higher the rates are, and the higher the code weights are, the higher the QoSs. Note that these two code parameters have earlier been investigated in the literature separately under code length and weight restrictions. In this contribution, we strive to jointly generate an OOC set with arbitrary code length and/or code weight while preserving the required correlation properties of the strict OOC. This allows then the different codes to be assigned for different user classes. The code set construction is also flexible in such a way that the code length and weight can be selected arbitrarily as long as the number of desired codes in each class of users can be supported.

Assume that we have a $Q$-class system where each class is represented by the length, weight pairs $\left(N_{q}, W_{q}\right)$ with $N_{1} \leq \cdots \leq N_{q} \leq \cdots \leq N_{Q}$ and the number of users in the $q$ th class is $K_{q}$. The code set can be represented by $\mathbf{C}=$ $\left\{C_{1}, C_{2}, \ldots, C_{q}, \ldots, C_{Q}\right\}$, where $C_{q}=\left\{c_{1, q}, c_{2, q}, \ldots, c_{K_{q}, q}\right\}$ is the set of codes in class $q$, and each $c_{m, q}$ is the set of mark position representation of the $m$ th code in the $q$ th class. The total number of codes in the multiclass OOC code set is $K=\sum_{q=1}^{Q} K_{q}$ and the total set of allowed differences in class $q$ is $D_{q}=\left\{1,2, \ldots, N_{q}-1\right\}$. Associated with each code $c_{m, q}$ is a set of MPDs $d_{m, q}$ according to (4) with a length of $W_{q}\left(W_{q}-1\right)$. Then, the differences consumed by $K_{q}$ codes in class $q$ is denoted by $\hat{D}_{q}$ with the number of elements equal to $K_{q} W_{q}\left(W_{q}-1\right)$. In addition to that, the allowed difference set of the multiclass code is limited by the length of the longest code as $\mathbf{D}=\left\{1,2, \ldots, N_{Q}-1\right\}$. Let $\Delta_{q}$ denote the set of remaining differences after generating all $i$ th classes with $1 \leq$ $i \leq q$. Then, the MPD construction technique of the multiclass code set $\mathbf{C}$ is outlined as follows:

1) Initialize $\mathbf{D}=\left\{1,2, \ldots, N_{Q}-1\right\}$ (total set of allowed differences) and $N_{0}=1 ; \Delta_{0}=\Phi$.

2) Set $q=1$ (start with shortest code class).

3) Form $D_{q}=\left\{N_{q-1}, \ldots, N_{q}-1\right\}+\Delta_{q-1}$ (allowed differences for $q$ th class).

4) Construct $c_{m, q}$ for $m=1$ to $K_{q}$ (using random search, combinatorial techniques, etc. [17]).

5) Form the consumed set of differences $\hat{D}_{q}$ for class $q$ from $c_{m, q}$ using (4).

6) $\mathbf{D}=\mathbf{D}-\hat{D}_{q}$ (remove the consumed difference set of $q$ th class from the total difference set).

7) $q=q+1$ (next longer length class).

8) $q \leq Q$ ? No: go to step 3 .

It should be emphasized that the code generation presented here makes use of the existing techniques in finding $c_{m, q}$, which is a computationally demanding task for higher code weights when random search is applied. However, in the investigated media access scheme, the required codes are sparse, and their code weights are low. On the other hand, since the optical power loss in the currently available encoding technology (based mainly on power splitting and combining) grows as $10 \log (W) \mathrm{dB}$. Therefore, lower weight codes are only of practical importance. Furthermore, since the code set is assumed to be available before any real system implementation, the speed of the code generation algorithm is, hence, of minor importance.

The proof of existence of an optimal OOC $(N, W, 1)$ code set for general values of $N$ and $W$ is a prohibitive and mathematically complex problem. However, it has been considered 
TABLE I

THREE-Class STRICT MWSL-OOC CODE SET

\begin{tabular}{c||c||c}
\hline High QoS (1000,7) & Medium QoS (1000,5) & Low QoS (1000,3) \\
\hline \hline$[1,35,162,302,423,464,479]$ & {$[1,398,431,564,726],[1,105,415,590,876]$} & {$[1,624,990],[1,613,644]$} \\
{$[1,245,324,337,441,657,910]$} & {$[1,107,381,666,736],[1,84,568,628,784]$} & {$[1,287,412],[1,16,54]$} \\
{$[1,114,152,444,504,751,927]$} & {$[1,59,258,282,633],[1,121,467,719,809]$} & {$[1,111,728],[1,343,766]$} \\
{$[1,255,322,565,676,737,807]$} & {$[1,690,849,877,993],[1,166,205,216,647]$} & {$[1,443,771],[1,489,655]$} \\
{$[1,54,106,513,648,711,768]$} & {$[1,469,484,909,970],[1,284,397,504,796]$} & {$[1,41,582],[1,798,818]$} \\
{$[1,99,231,592,767,962,969]$} & {$[1,112,425,588,927],[1,507,600,748,926]$} & {$[1,524,906],[1,466,687]$} \\
{$[1,76,113,332,342,570,802]$} & {$[1,162,285,622,900],[1,50,238,864,866]$} & {$[1,124,203],[1,217,220]$} \\
{$[1,27,74,392,403,525,603]$} & {$[1,527,653,807,971],[1,100,159,543,844]$} & {$[1,82,549],[1,78,821]$} \\
& {$[1,291,446,753,896],[1,69,90,260,598]$} & {$[1,22,448],[1,525,925]$} \\
& {$[1,67,120,365,800],[1,863,873,905,945]$} & {$[1,786,964],[1,566,899]$} \\
\hline
\end{tabular}

for specific values of the code weight and length. For example, Ge and Yin [20] considered the special case of an OOC with $(N, 4,1)$ and concluded that in order to obtain an optimal OOC, the code length should be restricted to $6(\bmod 12)$ and $24(\bmod 48)$. Therefore, investigation of the optimality of cardinality for the MWML-OOC is beyond the scope of this paper. Alternatively, we consider only the upper bound on each class cardinality conditioned on the number of codes in the other classes. For a $Q$-class MWML-OOC with a given $\left(N_{q}, W_{q}\right)$, the upper bound on the $q$ th class cardinality is a function of $\left(N_{q}, W_{q}\right), K_{i}$, and $W_{i}$ for $i<q$. This is summarized in the following.

A $Q$-class strict MWML-OOC code set $\mathbf{C}$ is represented by the length and weight pairs $\left(N_{q}, W_{q}\right)$ where $N_{1} \leq N_{2} \leq \cdots \leq$ $N_{q} \leq \cdots \leq N_{Q}$. The cardinality $|\mathbf{C}|$ and the number of codes in the $q$ th class $K_{q}$ are given by

$$
|\mathbf{C}|=K=\sum_{q=1}^{Q} K_{q}
$$

subject to the constraint of

$$
\frac{\sum_{q=1}^{Q} K_{q} W_{q}\left(W_{q}-1\right)}{N_{Q}-1} \leqslant 1
$$

and

$$
K_{q} \leqslant\left\lfloor\frac{N_{q}-N_{q-1}+\Delta_{q-1}}{W_{q}\left(W_{q}-1\right)}\right\rfloor
$$

\section{Examples of Multiclass OOCs}

In this section, we consider three cases of a strict multiclass OOC by using multiple searches for each code set. It should be noted that repeated search for each multiclass code set results in multiple code sets with the same weight and length parameters. The availability of multiple multiclass OOC code sets with the same $\left(N_{q}, W_{q}\right)$ can be used to enhance the security of the system. This can be implemented by commanding the users to switch to other multiclass OOCs with the same parameters from time to time. For more on OCDMA security, refer to [21].
Case 1: Multiweight Single-Length OOC (MWSL-OOC): This is beneficial in systems with fixed data rates supporting different QoS, since the signal-to-interference ratio in optical networks employing $\mathrm{OOC}$ and perfect matched filtering is proportional to the square of the code weight [22]. We consider a three-class system with $N_{1}=N_{2}=N_{3}=N$ and $W_{1}>W_{2}>$ $W_{3}$ where $W_{1}$ represents the weight of highest QoS users in terms of BER performance, $W_{2}$ the medium QoS users, and $W_{3}$ the low QoS users. The number of supported codes in one class in a multiclass OOC is no longer bounded by (3) and is determined by the desired number of codes in the other classes according to (6) and (7).

Table I shows an example of a generated three-class MWSLOOC. The code set is characterized by $(1000,7),(1000,5)$, and $(1000,3)$ and satisfies the autocorrelation and cross correlation properties of the strict OOCs. For the $(1000,7)$ single-class OOC, the number of codes is upper bounded by 23 from (3), similarly, the $(1000,5)$ and $(1000,3)$ are upper bounded by 49 and 166, respectively. In this example, the number of high QoS and medium QoS codes is set to eight and 20, respectively, then from (6), the upper bound on the cardinality of the low QoS codes is 43 , of which only 20 are generated.

Case 2: Single-Weight Multilength OOC (SWML-OOC): This is applicable to systems with equal QoS and supporting variable data rate. In this example, we generated a three-class SWML-OOC listed in Table II with nine high rate, 20 medium rate, and 20 low rate codes. The tabulated three-class SWMLOOC code set is characterized by $(300,5)$ for the high rate users, $(1000,5)$ for the medium rate users, and $(1500,5)$ for the low rate users. Due to the orthogonality properties of the generated strict SWML-OOC set, the relative QoS of the classes (in terms of error rate performance) is almost independent of the length of codes. In other words, different length classes have the same error performance, furthermore, increasing the code length of any class decreases the error rate of all classes in the same proportion. This is verified in the numerical results subsection (Fig. 9).

Case 3: MWML-OOC: In this case, the weights and lengths of the multiclass OOC is selected arbitrarily. Table III lists a three-class MWML-OOC set. The selected parameters of the multiclass code is such that high rate users get high QoS and low rate users get low QoS. The set is characterized by $(550,7)$, $(930,5)$, and $(1300,3)$ for the high, medium, and low rate QoS, 
TABLE II

ThreE-Class Strict SWML-OOC Code SET

\begin{tabular}{c||c||c}
\hline High Rate (300,5) & Medium Rate (1000,5) & Low Rate (1500,5) \\
\hline \hline$[1,32,55,94,175]$ & {$[1,18,639,701,947],[1,338,737,916,964]$} & {$[1,31,806,1030,1274],[1,163,277,731,1317]$} \\
{$[1,45,95,128,135]$} & {$[1,93,204,788,875],[1,81,409,509,654]$} & {$[1,242,427,932,1350],[1,100,179,547,1200]$} \\
{$[1,108,113,119,165]$} & {$[1,34,418,441,559],[1,13,525,646,723]$} & {$[1,53,234,895,1434],[1,702,1027,1369,1381]$} \\
{$[1,150,182,225,297]$} & {$[1,74,125,372,845],[1,4,108,655,932]$} & {$[1,223,335,426,1314],[1,466,515,1304,1373]$} \\
{$[1,124,162,172,259]$} & {$[1,113,263,287,455],[1,188,384,451,770]$} & {$[1,149,746,929,1084],[1,703,1158,1238,1444]$} \\
{$[1,102,147,160,161]$} & {$[1,431,432,595,641],[1,354,665,669,685]$} & {$[1,294,910,1073,1483],[1,112,278,328,888]$} \\
{$[1,36,61,114,231]$} & {$[1,143,435,846,899],[1,20,250,780,815]$} & {$[1,108,458,1222,1269],[1,677,778,872,1179]$} \\
{$[1,129,206,235,298]$} & {$[1,75,470,694,993],[1,148,336,427,505]$} & {$[1,967,1107,1111,1142],[1,52,640,1082,1104]$} \\
{$[1,31,115,117,133]$} & {$[1,415,717,853,992],[1,183,244,829,831]$} & {$[1,349,747,957,982],[1,189,883,956,1459]$} \\
& {$[1,129,224,289,768],[1,297,376,534,800]$} & {$[1,698,715,1144,1403],[1,170,213,528,586]$} \\
\hline
\end{tabular}

TABLE III

Three-Class Strict MWML-OOC Code Set

\begin{tabular}{c||c||c}
\hline \multicolumn{1}{c||}{ High Rate High QoS (550,7) } & Medium Rate Medium QoS (930,5) & Low Rate Low QoS (1300,3) \\
\hline \hline$[1,283,292,313,431,439,479]$ & {$[1,50,381,409,627],[1,6,536,698,773]$} & {$[1,499,1275],[1,362,378]$} \\
{$[1,4,63,153,222,223,247]$} & {$[1,89,357,364,640],[1,37,137,210,891]$} & {$[1,1059,1299],[1,761,1123]$} \\
{$[1,5,214,315,331,446,541]$} & {$[1,67,463,534,791],[1,283,579,852,853]$} & {$[1,297,1271],[1,671,1232]$} \\
{$[1,138,317,396,424,488,495]$} & {$[1,173,403,510,557],[1,45,186,741,871]$} & {$[1,136,764],[1,73,334]$} \\
& {$[1,118,127,221,564],[1,216,307,797,839]$} & {$[1,34,509],[1,962,1107]$} \\
& {$[1,492,601,772,904],[1,5,25,257,751]$} & {$[1,214,616],[1,326,1028]$} \\
& {$[1,334,762,787,826],[1,198,306,335,386]$} & {$[1,15,1018],[1,522,1206]$} \\
& {$[1,201,211,652,684],[1,423,545,804,834]$} & {$[1,41,232],[1,142,496]$} \\
& {$[1,227,279,641,724],[1,36,427,621,771]$} & {$[1,153,675],[1,86,1298]$} \\
& {$[1,502,561,750,878],[1,358,473,696,923]$} & {$[1,49,422],[1,285,506]$} \\
\hline
\end{tabular}

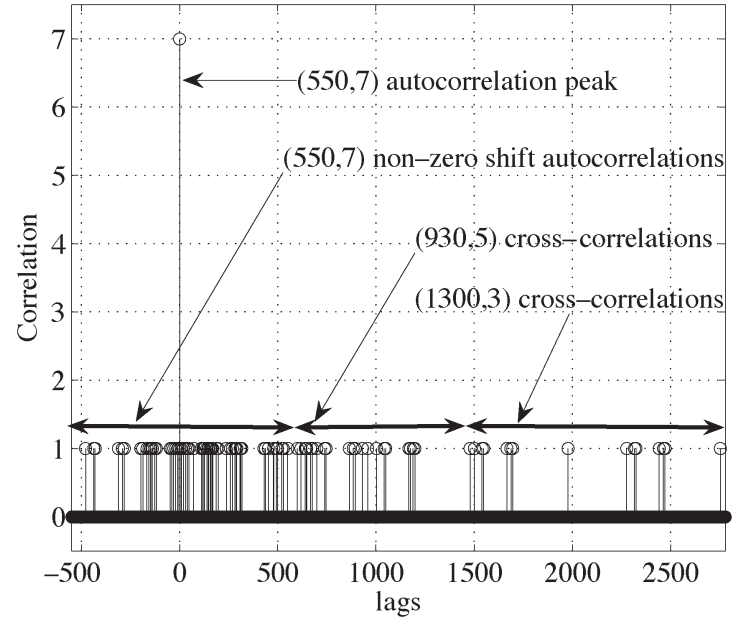

Fig. 3. Autocorrelation and cross correlation values for the three-class MWML-OOC example.

respectively. Note that long code lengths are not restricted to be an integer multiple of short codes but can be arbitrarily selected. It can be simply verified that the nonzero shift autocorrelation peak is one, and the cross correlation between any two codes is bounded by one. This is demonstrated in Fig. 3 for the first code in the high QoS class as the desired user and then applying the desired user code, the first code in the medium, and the first code in the low QoS classes to the correlator sequentially.

\section{Performance Analysis}

In asynchronous $K$-user CDMA systems, the received signal is the sum of the desired user signal and the signals of the other $K-1$ interfering users each with its own delay time. The time delay offsets are assumed to be a multiple of the chip period, which is known as the chip synchronous scenario that results in the upper bound on the bit error performance [7]. Let us assume the condition of perfect power balance (i.e., received power of all users is equal), and let us neglect other noise sources, such as the thermal noise from receiver's electronics, or shot noise from the photodetector. Furthermore, in our analysis, we did not account for the photon counting nature during the photodetection process. Different approaches to account for the photon arrival rate are used in the literature such as large deviations theory and saddlepoint approximations [23]-[25]. In what follows, we consider only the case of the strict MWML-OOC code set who have, by definition, nonzero shift autocorrelation and cross correlation properties bounded by one.

\section{A. System Description}

In order to evaluate the bit error probability performance of the strict MWML-OOC-based optical network, the decision variable at the output of the decorrelator is written as the desired signal-plus-interference form. We assume a $Q$-class system, with different code lengths such that 


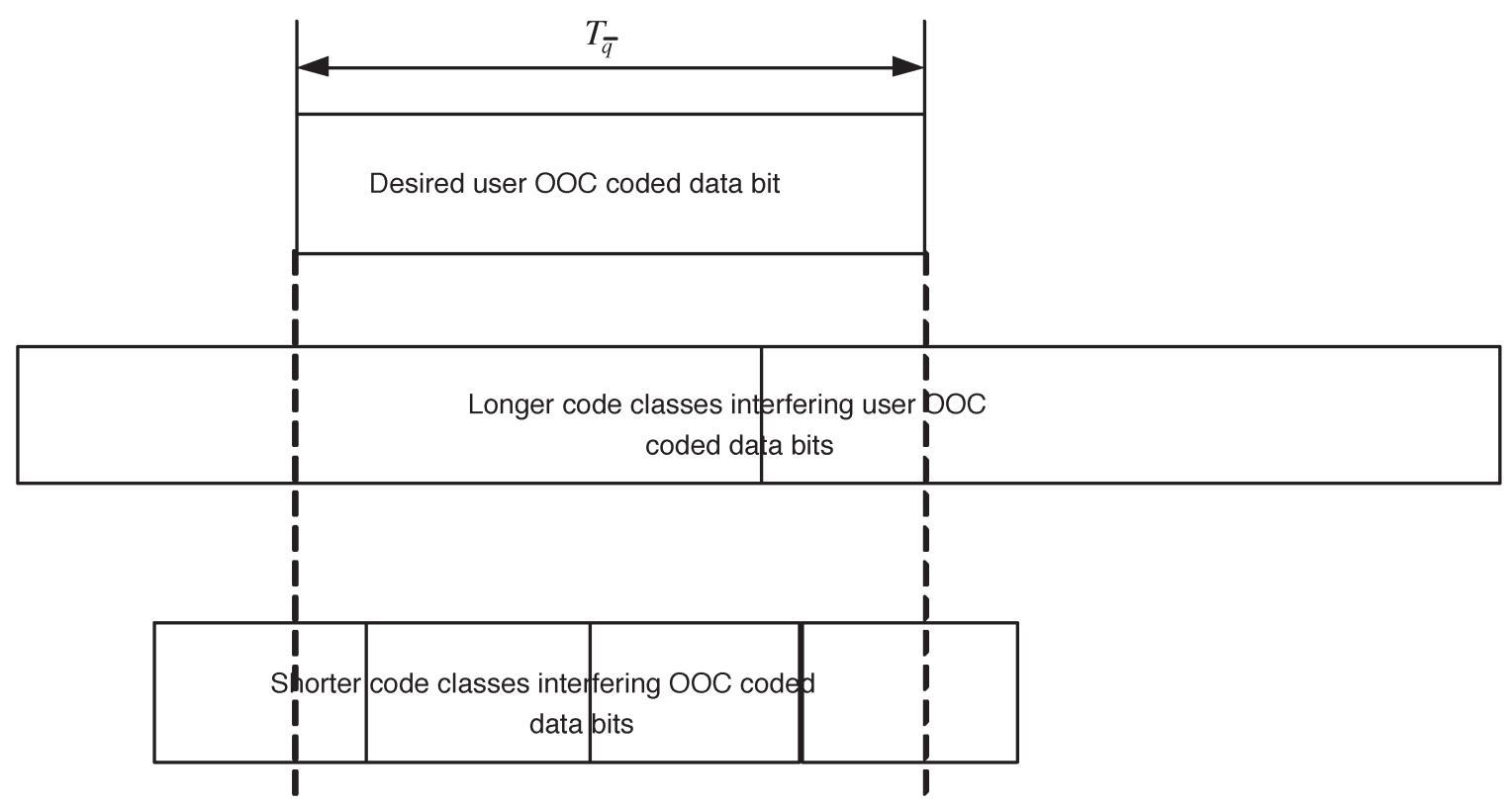

Fig. 4. Longer and shorter classes interference.

$N_{1} \leqslant N_{2} \leqslant \cdots \leqslant N_{q} \leqslant \cdots \leqslant N_{Q}$ and arbitrary weights. The number of users in each class is $K_{q}, q \in\{1,2, \ldots, Q\}$. Without loss of generality, we assume that the desired user is the first user in the class indexed $\bar{q}$. Then, the binary data signal of the $k$ th user in the $q$ th class is denoted by $b_{k, q}(t)=\sum_{i=-\infty}^{\infty} b_{i, k, q} p_{T_{q}}\left(t-i T_{q}\right)$, where $b_{i, k, q} \in\{0,1\}$ is the $i$ th binary data, and $p_{T_{q}}(t)$ is a rectangular pulse starting at zero and of width $T_{q}$. Likewise, the signature waveform of the $k$ th user in the $q$ th class can be written as $c_{k, q}(t)=$ $\sum_{j=-\infty}^{\infty} c_{j, k, q} p_{T_{c}}\left(t-j T_{c}\right)$, where $c_{j, k, q} \in\{0,1\}$ is the $j$ th chip in the code sequence and satisfying $c_{j+N_{q}, k, q}=c_{j, k, q}$. The chip duration of all classes is the same and given by $T_{c}=T_{q} / N_{q}$. Then, the intensity-modulated optical CDMA signal is given by $s_{k, q}(t)=c_{k, q}(t) b_{k, q}(t)$, where we assumed that the received intensity level for all users is normalized to unity in all classes. After passing the network, each user's optical CDMA signal will arrive to the receivers along with the desired signal giving rise to MAI. The MAI signals will arrive at different delays uniformly distributed over $\tau_{k, q} \in\left[0, T_{q}\right)$, and the desired signal delay is assumed to be zero, i.e., $\tau_{1, \bar{q}}=0$. Then, the received signal at the decorrelator input can be written as

$$
\begin{aligned}
r(t) & =\sum_{q=1}^{Q} \sum_{k=1}^{K_{q}} s_{k, q}\left(t-\tau_{k, q}\right) \\
& =s_{1, \bar{q}}(t)+r_{(q \geqslant \bar{q})}(t)+r_{(q<\bar{q})}(t) .
\end{aligned}
$$

In what follows, all summing operations indexed by $k$ are evaluated such that $k \neq 1$ if $q=\bar{q}$, i.e., the desired user in the desired class is excluded from the summations. In (8), we have

$$
r_{(q \geqslant \bar{q})}(t)=\sum_{q=\bar{q}}^{Q} \sum_{k=1}^{K_{q}} s_{k, q}\left(t-\tau_{k, q}\right)
$$

which is the received signal from users in equal and longer code length classes, and

$$
r_{(q<\bar{q})}(t)=\sum_{q=1}^{\bar{q}-1} \sum_{k=1}^{K_{q}} s_{k, q}\left(t-\tau_{k, q}\right)
$$

which is the received signal from shorter code length classes. Without loss of generality, we consider the effect of MAI on the detection of data bit $b_{0,1, \bar{q}}$. Then, the correlator output is given by

$$
\begin{aligned}
Z_{0,1, \bar{q}} & =\int_{0}^{T_{\bar{q}}} r(t) c_{1, \bar{q}}(t) d t \\
& =T_{c} W_{\bar{q}} b_{0,1, \bar{q}}+\sum_{q=\bar{q}}^{Q} \sum_{k=1}^{K_{q}} I_{(k, q \geqslant \bar{q})}^{1, \bar{q}}+\sum_{q=1}^{\bar{q}-1} \sum_{k=1}^{K_{q}} I_{(k, q<\bar{q})}^{1, \bar{q}}
\end{aligned}
$$

where $I_{(k, q \geqslant \bar{q})}^{1, \bar{q}}$ is the interference from equal and longer code length users, and $I_{(k, q<\bar{q})}^{1, \bar{q}}$ is the interference due to shorter code length classes. As demonstrated in Fig. 4, the desired user data bit will be hit by at most two interfering data bits from users in classes with $(q \geqslant \bar{q})$ and at most $s+2$ interfering data bits from users in classes with $(q<\bar{q})$, where $s=\left\lfloor T_{\bar{q}} / T_{q}\right\rfloor$. Generally, the interference terms for the $m$ th user in the $\bar{q}$ th class can be evaluated from

$$
\begin{aligned}
I_{(k, q \geqslant \bar{q})}^{m, \bar{q}}=b_{-1, k, q} & \int_{0}^{\tau_{k, q}} c_{k, q}\left(t-\tau_{k, q}\right) c_{m, \bar{q}}(t) d t \\
& +b_{0, k, q} \int_{\tau_{k, q}}^{T_{\bar{q}}} c_{k, q}\left(t-\tau_{k, q}\right) c_{m, \bar{q}}(t) d t
\end{aligned}
$$


and

$$
\begin{aligned}
I_{(k, q<\bar{q})}^{m, \bar{q}}= & b_{-1, k, q} \int_{0}^{\tau_{k, q}} c_{k, q}\left(t-\tau_{k, q}\right) c_{m, \bar{q}}(t) d t \\
& +b_{0, k, q} \int_{\tau_{k, q}}^{T_{q}+\tau_{k, q}} c_{k, q}\left(t-\tau_{k, q}\right) c_{m, \bar{q}}(t) d t \\
& +\cdots \\
& +b_{s-1, k, q} \int_{(s-1) T_{q}+\tau_{k, q}} c_{k, q}\left(t-\tau_{k, q}\right) c_{m, \bar{q}}(t) d t \\
& +b_{s, k, q} \int_{s T_{q}+\tau_{k, q}}^{s T_{q}+\tau_{k, q}} c_{k, q}\left(t-\tau_{k, q}\right) c_{m, \bar{q}}(t) d t
\end{aligned}
$$

Equation (9) can be rewritten as

$$
Z_{0,1, \bar{q}}=T_{c} W_{\bar{q}} b_{0,1, \bar{q}}+\sum_{q=\bar{q}}^{Q} I_{(q \geqslant \bar{q})}^{1, \bar{q}}+\sum_{q=1}^{\bar{q}-1} I_{(q<\bar{q})}^{1, \bar{q}}
$$

where $I_{(q \geqslant \bar{q})}^{1, \bar{q}}$ and $I_{(q<\bar{q})}^{1, \bar{q}}$ is the sum of interference due to $K_{q}$ users in $q \geqslant \bar{q}$ and $q<\bar{q}$ classes, respectively. Normalizing by $T_{c}$ and denoting the sum of the interference from $q$ th class users on a user in the $\bar{q}$ th class as $I_{q \bar{q}}$, we get

$$
\begin{aligned}
Z & =W_{\bar{q}} b_{0,1, \bar{q}}+\sum_{q=1}^{Q} I_{q \bar{q}} \\
& =W_{\bar{q}} b_{0,1, \bar{q}}+I_{\bar{q}}
\end{aligned}
$$

where $I_{\bar{q}}$ is the total MAI from all classes.

\section{B. BERs}

In the chip synchronous case, each chip position can be occupied by a mark from the desired and interfering user with some probability. Therefore, the sum of such possible interference will follow the binomial random variable distribution. Consequently, the random variables $I_{q \bar{q}}$ in (13) are binomially distributed with parameters $\left(K_{q}, p_{q \bar{q}}\right)$ and $K_{q}=K_{\bar{q}}-1$ when $q=\bar{q} \cdot p_{q \bar{q}}$ denotes the probability that a mark position from a code in class $\bar{q}$ is hit by a mark from a code in class $q$ and derived in Appendix

$$
p_{q \bar{q}}=\frac{W_{\bar{q}} W_{q}}{2 N_{q}} .
$$

Note that, in general, $p_{q \bar{q}} \neq p_{\bar{q} q}$. The factor of $1 / 2$ is included due to the equal probability for a user to send a " 1 " or a " 0 " data bit. A special case is the single class that gives $p=W^{2} / 2 N_{q}$ [7]. The multiclass hit probability in (15) depends inversely on the length of the interfering classes. Thus, long code classes cause lower interference and shorter code classes cause higher interference. The moment generating function of the binomially distributed random variables $I_{q \bar{q}}$ is given by [26]

$$
M_{I_{q \bar{q}}}(t)=E\left[e^{t I_{q \bar{q}}}\right]=\left(\left(1-p_{q \bar{q}}\right)+p_{q \bar{q}} e^{t}\right)^{K_{q}} .
$$

Since the random variables $I_{q \bar{q}}$ are independent, then the moment-generating function of the total interference can be expressed as

$$
M_{I_{\bar{q}}}(t)=\prod_{q=1}^{Q} M_{I_{q \bar{q}}}(t)
$$

then the mean of the MAI is

$$
R=\mathbf{E}\left[I_{\bar{q}}\right]=M_{I_{\bar{q}}}^{\prime}(0)=\sum_{q=1}^{Q} K_{q} p_{q \bar{q}}
$$

and the mean square value is

$$
\begin{aligned}
\mathbf{E}\left[\left(I_{\bar{q}}\right)^{2}\right] & =M_{I_{\bar{q}}}^{\prime \prime}(0) \\
& =\left(\sum_{q=1}^{Q} K_{q} p_{q \bar{q}}\right)^{2}+\sum_{q=1}^{Q} K_{q} p_{q \bar{q}}\left(1-p_{q \bar{q}}\right) .
\end{aligned}
$$

Therefore, the variance of the MAI is given by

$$
\sigma_{I_{\bar{q}}}^{2}=\sum_{q=1}^{Q} K_{q} p_{q \bar{q}}\left(1-p_{q \bar{q}}\right) .
$$

For the single-class system, the variance is given by $\sigma^{2}=$ $(K-1) p(1-p)$ [7]. Since the OOCs are very sparse with $W / N \ll 1$, the binomially distributed $I_{q \bar{q}}$ can be approximated by a Poisson distribution for large $K_{q}$ and small $p_{q \bar{q}}$ as

$$
P_{I_{q \bar{q}}}\left(I_{q \bar{q}}=n\right) \approx \frac{\left(K_{q} p_{q \bar{q}}\right)^{n}}{n !} e^{-K_{q} p_{q \bar{q}}} .
$$

Since the sum of independent identically distributed (i.i.d.) Poisson distributed random variables is also Poisson distributed with PMF, then

$$
\begin{aligned}
P_{I_{\bar{q}}}\left(I_{\bar{q}}=n\right) & \approx \frac{\left(\sum_{q=1}^{Q} K_{q} p_{q \bar{q}}\right)^{n}}{n !} e^{-\sum_{q=1}^{Q} K_{q} p_{q \bar{q}}} \\
& =\frac{R^{n}}{n !} e^{-R} .
\end{aligned}
$$

The bit error probability (BER) of the multiclass OOC system can be evaluated from the decision variable $Z$ by

$$
\begin{aligned}
P(E) & =\frac{1}{2}\left[P\left(Z \geqslant \mu \mid b_{0,1, \bar{q}}=0\right)+P\left(Z<\mu \mid b_{0,1, \bar{q}}=1\right)\right] \\
& =\frac{1}{2} P\left(I_{\bar{q}} \geqslant \mu \mid b_{0,1, \bar{q}}=0\right)
\end{aligned}
$$

where $\mu$ is the threshold of the decision device. The second term is set to zero due to the positivity assumption, and the factor of 


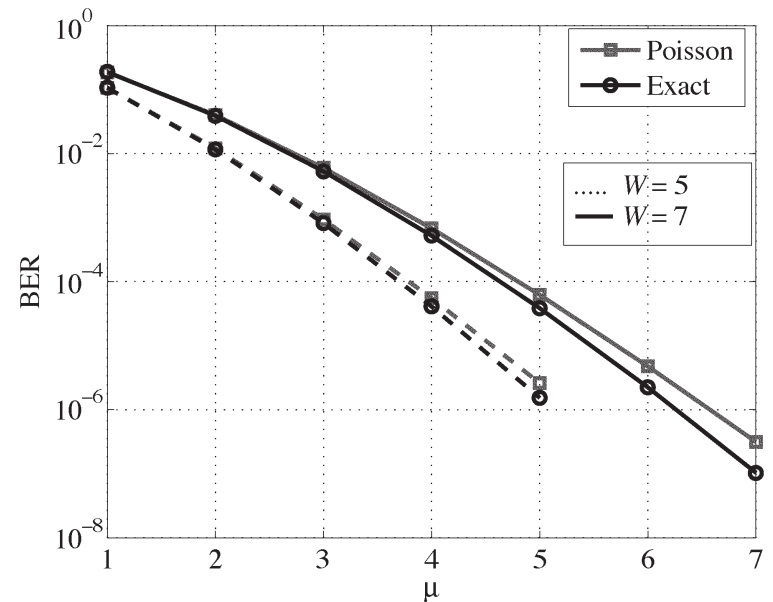

Fig. 5. Exact BER upper bound and its Poisson distribution approximation with 20 user OOCs as a function of the threshold. $N=1000$ and $W=5,7$.

$1 / 2$ is due to the equal likelihood of transmitting " 0 " and " 1 " data bits. It is simple to show that the BER is given by

$$
P(E) \approx \frac{1}{2}\left[1-e^{-R} \sum_{n=0}^{\mu-1} \frac{R^{n}}{n !}\right] .
$$

The error probability can be written in terms of the incomplete gamma function $[27$, p. 218$]$ as

$$
\Gamma(n, x) \triangleq \int_{0}^{x} e^{-t} t^{n-1} d t
$$

by using the identity

$$
\sum_{n=c}^{\infty} \frac{x^{n}}{n !} e^{-x}=\frac{\Gamma(c, x)}{\Gamma(c, \infty)}
$$

Then, (24) can be written compactly as

$$
P(E)=\frac{1}{2} \frac{\Gamma(\mu, R)}{\Gamma(\mu, \infty)}
$$

Thus, the bit error probability is one half the ratio of the incomplete gamma function with the threshold and the mean of the MAI as parameters to the incomplete gamma function with the threshold and infinity as parameters. This form of the error probability is a convenient functional form, although (24) is simpler for numerical evaluation. Fig. 5 shows the exact BER performance of the single-class OOC system based on binomial distribution [7], and the approximated BER based on Poisson distribution of (24), with the code length of 1000 , number of users of 20, and the code weight of 5 or 7 . It is clear that the Poisson approximation results in acceptable accuracy and provides a relatively simple formula that simplifies the evaluation of the BER for a multiclass OOC optical CDMA system.

\section{Numerical Results}

Using (24), the influence of the multiclass OOC code parameters on the performance is investigated. Consider a

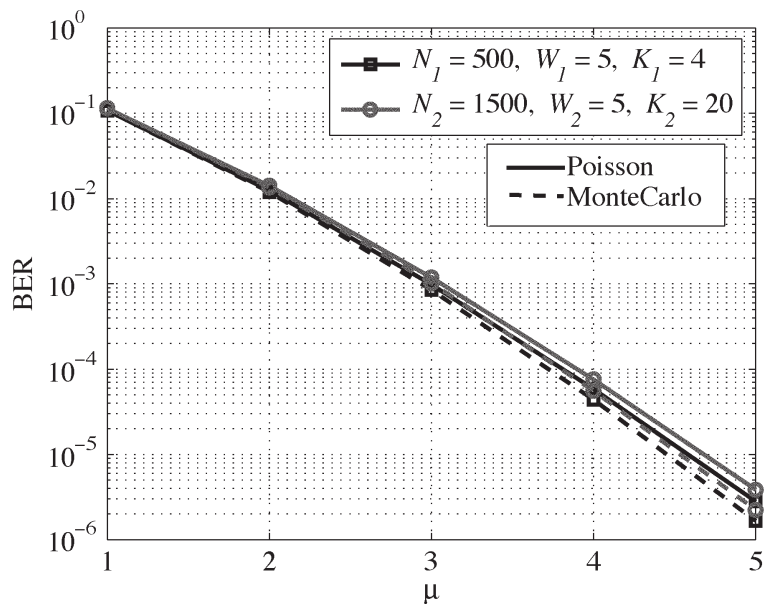

Fig. 6. BER for a two-class OOC system with equal weights and different lengths. Dotted lines are the Monte Carlo simulation results.

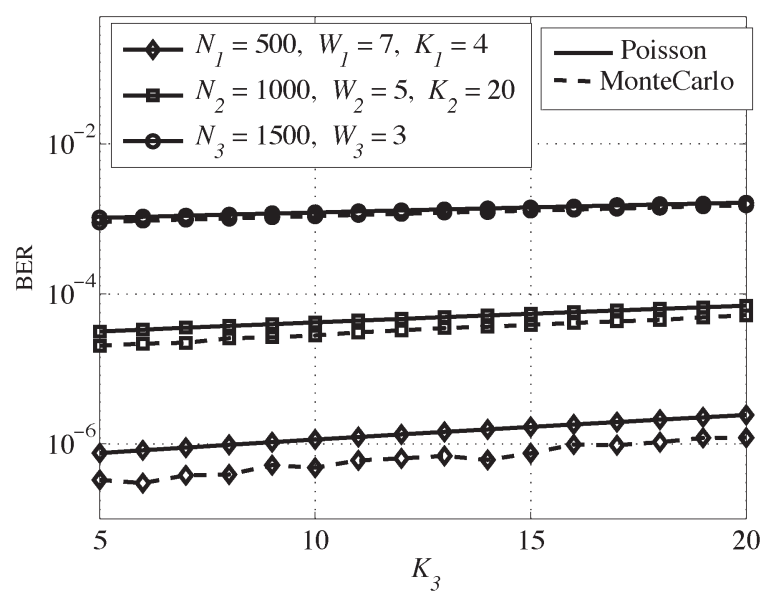

Fig. 7. BER for a three-class OOC, the number of users in the third class is varied from 5 to 20. Dotted lines are the Monte Carlo simulation results.

two-class SWML-OOC system with four users in the high rate class $(500,5)$ and 20 users in the low rate class $(1500,5)$. The BER is plotted for both user classes in Fig. 6 with virtually the same performance because of equal weight and orthogonality requirements. A three-class MWML-OOC performance is shown in Fig. 7. The three classes are defined by $(500,7)$, $(1000,5)$, and $(1500,3)$. The longer code (lower rate) class is inferior to the other classes due to its lower weight. In addition, increasing the number of users in the longer code class has small effect on performance because of low probability of hit.

In Fig. 8, a two-class OOC system performance is plotted for both classes with a code length of the low rate class double that of the high rate class, namely, $N_{1}=1000$ and $N_{2}=2000$. The code weight of the high rate class is fixed at the value of 5 and the code weight of the low weight class is varied from 4 to 9 . It is clear that the performance of one class is better than the other whenever its code weight is higher, with approximately equal performance at the two curves crossing point of 5 (i.e., equal weight). It is noticed that the longer code class has a slightly higher BER than the shorter code class. This is mainly due to the increased hit probability for the shorter codes than the longer codes according to (15). Although the 


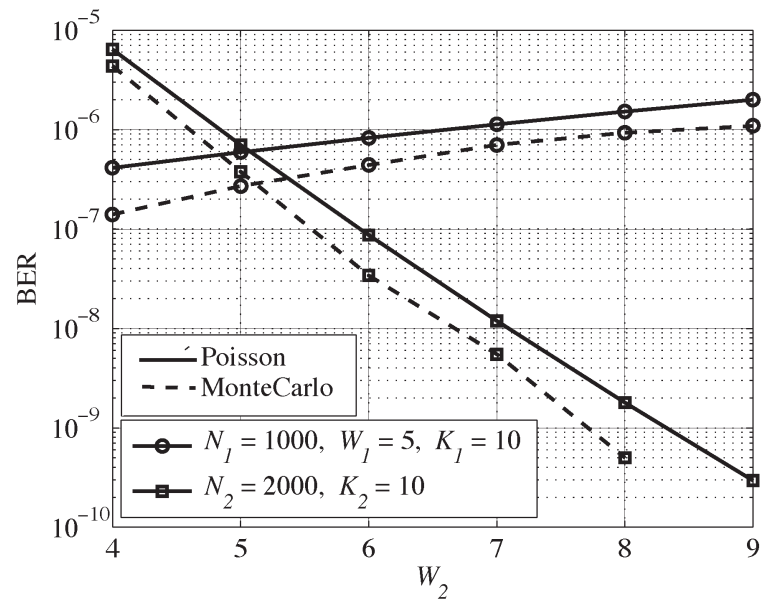

Fig. 8. BER for a two-class OOC system as a function of the code weight of the low rate class. Dotted lines are the Monte Carlo simulation results.

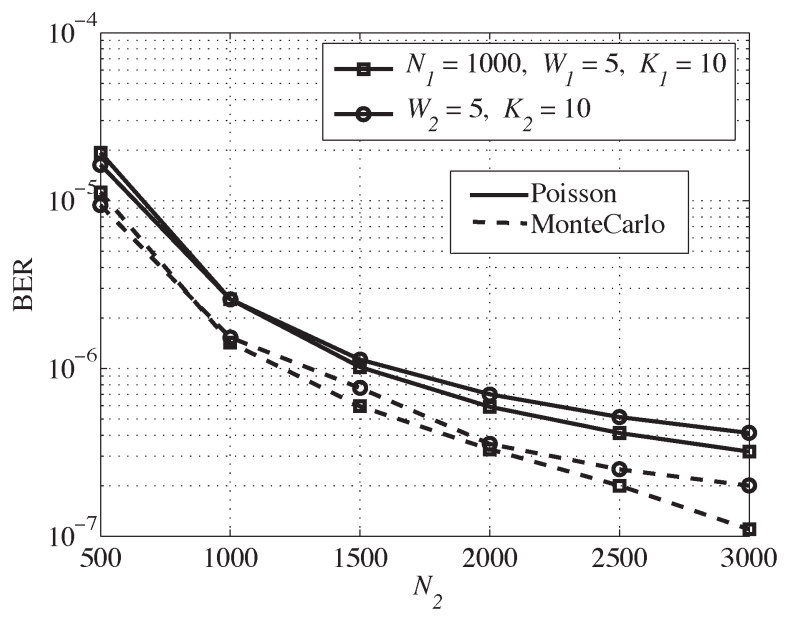

Fig. 9. BER for a two-class OOC system as a function of the code length of the second class. Dotted lines are the Monte Carlo simulation results.

weight of the high rate class need not to be less than that of the lower rate user classes, higher weight will limit the number of supported users in the high rate class significantly due to the shorter code length. Finally, the effect of the code length in one class when fixing all the other parameters is depicted in Fig. 9. As the code length of one of the classes increases, its MAI on all the other classes decreases because of decreasing probability of hit. Thus, increasing (decreasing) the code length of one class will decrease (increase) the BER of all the other classes in a same proportion. In order to validate the Poisson approximation applied to the MAI, we performed intensive Monte Carlo simulations of the multiclass cases presented above. Around $10^{9}$ trials are performed in each case to get an acceptable BER estimate. In all cases, the MAI Poisson approximation overestimates the BER slightly. Therefore, by applying the MAI Poisson assumption, we are on the conservative side, and the exact BER is always lower than the estimated value. Moreover, the BER analysis of the multiclass OOC system is simplified significantly by using the Poisson approximation with an acceptable accuracy specially for the long-length low-weight OOCs.

\section{CONCLUSION}

We have presented in this paper a proposal of the construction of a multiweight multilength strictly orthogonal optical code set. The code set is flexible, because it can be designed for any code lengths and weights limited only by the possible number of codes. Moreover, the code set contains codes with different code lengths and code weights while satisfying the required correlation properties of the strict OOCs. The different code lengths support data rate differentiation, and the different code weights support QoS differentiation. The presented technique is interesting from a practical point of view, since the hardware requirement is almost the same as that we have for the fixed-length fixed-weight OOC except for mature optical switches added to the encoders and decoders. We have considered a multiclass system, with different code lengths and weights, and demonstrated how the multilength multiweight strict OOCs can provide the proposed QoS differentiation. By approximating the binomially distributed MAI per class using the Poisson distribution, the analysis of multiclass MAI is simplified significantly. We showed that the multiclass OOC performance differentiation is controlled mainly by the code weights. By changing the length of any class, the performance of all classes changes by the same amount. This is a direct consequence of the correlation properties of the strict OOC.

\section{APPENDIX \\ MWML-OOC HIT PROBABILITY}

In this appendix, we derive the probability of hit for multiclass OOC given in (15). All summations indexed by $k$ are evaluated such that $k \neq 1$ if $q=\bar{q}$. The random variables in $(12), I_{(q \geqslant \bar{q})}^{1, \bar{q}}$ and $I_{(q<\bar{q})}^{1, \bar{q}}$ are binomially distributed with a mean value of

$$
\mathbf{E}\left[I_{(q \geqslant \bar{q})}^{1, \bar{q}}\right]=\mathbf{E}\left[I_{(q<\bar{q})}^{1, \bar{q}}\right]=T_{c} K_{q} p_{q \bar{q}}
$$

where $p_{q \bar{q}}$ is the probability that a mark from a user in class $q$ coincides with a mark for a user in class $\bar{q}$. To find these probabilities, we make use of (10) and (11) as

$$
\mathbf{E}\left[I_{(q \geqslant \bar{q})}^{1, \bar{q}}\right]=\mathbf{E}\left[\sum_{k=1}^{K_{q}} I_{(k, q \geqslant \bar{q})}^{1, \bar{q}}\right]=\sum_{k=1}^{K_{q}} \mathbf{E}\left[I_{(k, q \geqslant \bar{q})}^{1, \bar{q}}\right] .
$$

To simplify, we define the integrations

$$
I_{\left(0 ; \tau_{k, q}\right)}=\int_{0}^{\tau_{k, q}} c_{k, q}\left(t-\tau_{k, q}\right) c_{m, \bar{q}}(t) d t
$$

and

$$
I_{\left(\tau_{k, q} ; T_{\bar{q}}\right)}=\int_{\tau_{k, q}}^{T_{\bar{q}}} c_{k, q}\left(t-\tau_{k, q}\right) c_{m, \bar{q}}(t) d t .
$$


Then, (A.2) can be detailed as

$$
\begin{array}{r}
\mathbf{E}\left[I_{(q \geqslant \bar{q})}^{1, \bar{q}}\right]=\frac{1}{2} \sum_{k=1}^{K_{q}}\left\{\mathbf{E}\left[I_{\left(0 ; \tau_{k, q}\right)}\right]+\mathbf{E}\left[I_{\left(\tau_{k, q} ; T_{\bar{q}}\right)}\right]\right\} \\
=\frac{1}{2} \sum_{k=1}^{K_{q}}\left\{\mathbf{E}_{\tau_{k, q}}\left[\mathbf{E}_{c_{\tau, q} \mid \tau_{k, q}}\left[I_{\left(0 ; \tau_{k, q}\right)}\right]\right]\right. \\
\left.+\mathbf{E}_{\tau_{k, q}}\left[\mathbf{E}_{c_{\tau, q} \mid \tau_{k, q}}\left[I_{\left(\tau_{k, q} ; T_{\bar{q}}\right)}\right]\right]\right\}
\end{array}
$$

where $\mathbf{E}_{X}[\bullet]$ is the expectation over the random variable $X$ and $\mathbf{E}_{X \mid Y}[\bullet]$ is the conditional expectation over the random variable $X$ given $Y$. In (A.5), we need the expectation $\mathbf{E}_{c_{\tau, q} \mid \tau_{k, q}}\left[I_{\left(0 ; \tau_{k, q}\right)}\right]$, which can be evaluated using the assumption of independent codes for each user

$$
\begin{aligned}
\mathbf{E}_{c_{\tau, q} \mid \tau_{k, q}} & {\left[\int_{0}^{\tau_{k, q}} c_{k, q}\left(t-\tau_{k, q}\right) c_{m, \bar{q}}(t) d t\right] } \\
= & \int_{0}^{\tau_{k, q}} \mathbf{E}_{c_{\tau, q} \mid \tau_{k, q}}\left[c_{k, q}\left(t-\tau_{k, q}\right)\right] \mathbf{E}_{c_{\tau, q} \mid \tau_{k, q}}\left[c_{m, \bar{q}}(t)\right] d t .
\end{aligned}
$$

From the signature signals, this can be reduced to

$$
\mathbf{E}_{c_{\tau, q} \mid \tau_{k, q}}\left[I_{\left(0 ; \tau_{k, q}\right)}\right]=\frac{W_{\bar{q}} W_{q}}{N_{\bar{q}} N_{q}} \tau_{k, q}
$$

Then

$$
\mathbf{E}_{\tau_{k, q}}\left[\mathbf{E}_{c_{\tau, q} \mid \tau_{k, q}}\left[I_{\left(0 ; \tau_{k, q}\right)}\right]\right]=\frac{1}{2} \frac{W_{\bar{q}} W_{q}}{N_{\bar{q}}} T_{c} .
$$

Similarly, $\mathbf{E}_{c_{\tau, q} \mid \tau_{k, q}}\left[I_{\left(\tau_{k, q}, T_{\bar{q}}\right)}\right]$ can be evaluated as

$$
\begin{aligned}
& \mathbf{E}_{c_{\tau, q} \mid \tau_{k, q}}\left[\int_{\tau_{k, q}}^{T_{\bar{q}}} c_{k, q}\left(t-\tau_{k, q}\right) c_{m, \bar{q}}(t) d t\right] \\
& =\int_{\tau_{k, q}}^{T_{\bar{q}}} \mathbf{E}_{c_{\tau, q} \mid \tau_{k, q}}\left[c_{k, q}\left(t-\tau_{k, q}\right)\right] \mathbf{E}_{c_{\tau, q} \mid \tau_{k, q}}\left[c_{m, \bar{q}}(t)\right] d t \\
& =\frac{W_{\bar{q}} W_{q}}{N_{\bar{q}} N_{q}}\left(\tau_{k, q}+T_{\bar{q}}\right) .
\end{aligned}
$$

Then

$$
\mathbf{E}_{\tau_{k, q}}\left[\mathbf{E}_{c_{\tau, q} \mid \tau_{k, q}}\left[I_{\left(\tau_{k, q} ; T_{\bar{q}}\right)}\right]\right]=\frac{W_{\bar{q}} W_{q}}{N_{\bar{q}} N_{q}} T_{c}\left(N_{\bar{q}}-\frac{N_{q}}{2}\right) .
$$

Using (A.6) and (A.7) in (A.5), we get

$$
\mathbf{E}\left[I_{(q \geqslant \bar{q})}^{1, \bar{q}}\right]=\frac{1}{2} \frac{W_{\bar{q}} W_{q}}{N_{q}} T_{c} K_{q} .
$$

Thus, from (A.8) and (A.1), the probability of hit is

$$
p_{q \bar{q}}=\frac{1}{2} \frac{W_{\bar{q}} W_{q}}{N_{q}}, \quad q \geqslant \bar{q} .
$$

A similar argument can be followed for the hit probability from users in classes $(q<\bar{q})$, which will give the same result as in (A.9). Thus, (15) is valid for any $q$.

\section{ACKNOWLEDGMENT}

The first author thanks H. El-Sallabi for his valuable discussions and comments. The first author also thanks Prof. $\mathrm{H}$. Shalaby for his reading and comments on the manuscript.

\section{REFERENCES}

[1] I. V. D. Voorde and G. V. D. Plas, "Full service optical access networks: ATM transport on passive optical networks," IEEE Commun Mag., vol. 35, no. 4, pp. 70-75, Apr. 1997.

[2] D. S. Burpee and P. W. Shumate, "Emerging residential broadband telecommunications," Proc. IEEE, vol. 82, no. 4, pp. 604-614, Apr. 1994.

[3] L. G. Kazovsky, T. Fong, and T. Hofmeister, "Optical local area network technologies," IEEE Commun. Mag., vol. 32, no. 12, pp. 50-54, Dec. 1994.

[4] G. Kramer, B. Mukherjee, and A. Maislos, Ethernet Passive Optical Network (EPON): A Missing Link in an End-to-End Optical Internet, S. Dixit, Ed. New York: Wiley, Mar. 2003.

[5] C. M. Assi, Y. Ye, S. Dixit, and M. A. Ali, "Dynamic bandwidth allocation for quality-of-service over Ethernet PONs," IEEE J. Sel. Areas Commun., vol. 21, no. 9, pp. 1467-1477, Nov. 2003.

[6] M. J. Parham, C. Smythe, and B. L. Weiss, "Code division multiple access techniques for use in optical fiber local area networks," Elect. Commun. J., vol. 4, no. 4, pp. 203-212, Aug. 1992.

[7] J. Salehi, "Code division multiple-access techniques in optical fiber networks-Part I: Fundamental concepts," IEEE Trans. Commun., vol. 37, no. 8, pp. 824-833, Aug. 1989.

[8] V. Baby, B. C. Wang, L. Xu, I. Glesk, and P. R. Prucnal, "Highly scalable serial-parallel optical delay line," Opt. Commun., vol. 218, no. 4-6, pp. 235-242, Apr. 2003.

[9] S. V Maric, O. Moreno, and C. J. Corrada, "Multimedia transmission in fiber-optic LANs using optical CDMA," J. Lightw. Technol., vol. 14, no. 10 , pp. 2149-2153, Oct. 1996.

[10] S. V. Maric and V. K. Lau, "Multirate fiber-optic CDMA: System design and performance analysis," J. Lightw. Technol., vol. 16, no. 1, pp. 9-17, Jan. 1998.

[11] E. Intay, H. M. H. Shalaby, P. Fortier, and L. A. Rusch, "Multirate optical fast frequency-hopping CDMA system using power control," J. Lightw. Technol., vol. 20, no. 2, pp. 166-177, Feb. 2002.

[12] W. C. Kowng and G.-C. Yang, "Double-weight signature pattern codes for multicore-fiber code-division multiple-access networks," IEEE Commun. Lett., vol. 5, no. 5, pp. 203-205, May 2001.

[13] F.-R. Gu and J. Wu, "Construction and performance analysis of variableweight optical orthogonal codes for asynchronous optical CDMA systems," J. Lightw. Technol., vol. 23, no. 2, pp. 740-748, Feb. 2005.

[14] W. C. Kowng and G.-C. Yang, "Design of multilength optical orthogonal codes for optical CDMA multimedia networks," IEEE Trans. Commun., vol. 50, no. 8, pp. 1258-1265, Aug. 2002.

[15] J.-G. Zhang, "Variable-bit-rate video transmission systems using optical fiber code-division multiplexing scheme," IEEE Trans. Consum. Electron., vol. 42, no. 4, pp. 874-884, Nov. 1996.

[16] N. G. Tarhuni and T. O. Korhonen, "Multi-weight multi-length strict optical orthogonal codes," in Proc. 6th Nordic Signal Processing Symp. (NORSIG), Espoo, Finland, Jun. 9-11, 2004, pp. 161-164.

[17] F. R. Chang, J. Salehi, and V. K. Wei, "Optical orthogonal codes: Design, analysis, and applications," IEEE Trans. Inf. Theory, vol. 35, no. 3, pp. 595-604, May 1989.

[18] J. Zhang, "Application of strict optical orthogonal codes to asynchronous all-optical CDMA networks," in Proc. Int. Conf. Communications Technology, Beijing, China, Oct. 22-24, 1998, pp. S35-06-1-S35-06-5.

[19] G.-C. Yang and W. C. Kwong, "Two dimensional spatial signature patterns," IEEE Trans. Commun., vol. 44, no. 2, pp. 184-191, Feb. 1996.

[20] G. Ge and J. Yin, "Constructions for optimal (v, 4, 1) optical orthogonal codes," IEEE Trans. Inf. Theory, vol. 47, no. 7, pp. 2998-3004, Nov. 2001.

[21] T. H. Shake, "Security performance of optical CDMA against eavesdropping," J. Lightw. Technol., vol. 23, no. 2, pp. 655-670, Feb. 2005.

[22] J. Salehi and C. A. Brackett, "Code division multiple-access techniques in optical fiber networks-Part II: Performance analysis," IEEE Trans. Commun., vol. 37, no. 8, pp. 834-842, Aug. 1989 
[23] M. B. Pearce and B. Aazhang, "Performance analysis of single-user and multiuser detectors for optical code division multiple access communication systems," IEEE Trans. Commun., vol. 43, no. 2-4, pp. 435-444, Feb.-Apr. 1995.

[24] L. B. Nelson and H. V. Poor, "Performance of multiuser detection for optical CDMA-Part I: Error probabilities," IEEE Trans. Commun., vol. 43, no. 11, pp. 2803-2811, Nov. 1995.

[25] T. K. Tang and K. B. Letaief, "Bit-error rate computation of optical CDMA communication systems by large deviations theory," IEEE Trans. Commun., vol. 46, no. 11, pp. 1422-1428, Nov. 1998.

[26] H. P. Hsu, Theory and Problems of Probability, Random Variables, and Random Processes. New York: McGraw-Hill, 1997.

[27] R. M. Gagliardi and S. Karp, Optical Communications. New York: Wiley, 1976.

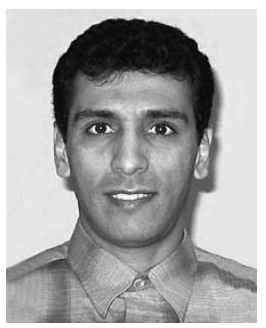

Naser G. Tarhuni (S'04) was born in Benghazi, Libya, on January 1970. He received the B.Sc. and M.Sc. degrees in electrical engineering from Garyounis University, Benghazi, in 1991 and 1996, respectively.

From 1994 to 2001, he worked as a Communications Engineer with the General Electricity Company of Libya (GECOL). During the period from 1997 to 2001, he also worked as a part-time Lecturer with the Electrical and Electronics Engineering Department, Garyounis University. Currently, he is a postgraduate student at the Helsinki University of Technology, Espoo, Finland. His research interests are in the area of multiple-access techniques in optical communications, optical signal processing, and wireless communications.

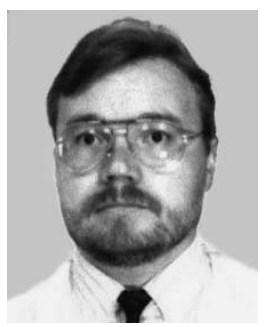

Timo O. Korhonen (S'89-M'91) received the Ph.D. degree in communications engineering from the Helsinki University of Technology (HUT), Espoo, Finland, in 1999.

He has been a Professor of telecommunications systems engineering with the Communications Laboratory, HUT, since 1999. His research interest include telecommunications technology in open system interconnection (OSI) levels $1-3$, especially media access control (MAC) and transmission aspects of orthogonal frequency-division multiplexing (OFDM) and code division multiple access (CDMA) systems, power line communications, fiber-optic communications, wideband radio propagation, fourthgeneration $(4 \mathrm{G})$ networks, and quality of service (QoS) issues. His current research interests include also topics in research of telecommunications product and service development process. He is an Editorial Board member of Kluwer's Journal of Wireless Personal Communications and has been working as a reviewer for several international scientific conferences.

Dr. Korhonen has worked as a Technical Program Committee Member/ Session Chair/Chair in several international conferences like the IEEE Conference on Communications (ICC), Global Telecommunications (GLOBECOM), Wireless Personal Mobile Communications (WPMC), and Finnish Wireless Communications Workshop (FWCW).

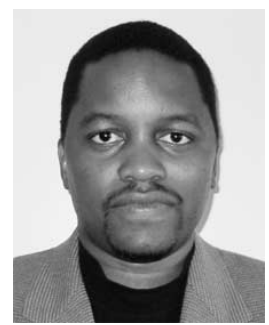

Edward Mutafungwa received the B.Eng. degree in electronic systems engineering and the M.Sc. degree in telecommunications and information systems from the University of Essex, Colchester, U.K., in 1996 and 1997, respectively, and the Dr.Sc.Tech. degree in communications engineering from the Helsinki University of Technology (HUT), Espoo, Finland, in 2004.

Since 1997, he has been lecturing and researching in various projects at the Communications Laboratory of HUT and has consulted for various companies. His research interests lie within the general fields of optical networking, network design, broadband wireless communications, intelligent transport systems, and intelligent computing.

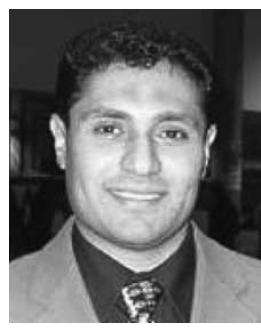

Mohammed S. Elmusrati (S'00-M'04) received the B.Sc. (with honors) and M.Sc. (with high honors) degrees in electrical engineering from the Electrical and Electronic Engineering Department, Garyounis University, Benghazi, Libya, in 1991 and 1995, respectively, and the Licentiate of Science in technology (with distinction) and the D.Sc. degree in control engineering from the Helsinki University of Technology (HUT), Espoo, Finland, in 2002 and 2004, respectively.

His research interests include radio resource management in wireless and optical communication, smart antennas, ultrawideband (UWB), wireless distributed actuator and sensor networks, and data fusion. He has received several grants from Garyounis University, Nokia Foundation, and The ELLA JA Georg Ehrnrootin Foundation. He is currently a full-time Lecturer at the Department of Computer Science, University of Vaasa, Vaasa, Finland, and a part-time Senior Researcher at the Automation Department, HUT. 